\title{
Proof-of-concept demonstration of free-form optics enhanced confocal Raman spectroscopy in combination with optofluidic lab-on-chip
}

\author{
Qing Liu ${ }^{1 *}$, Diane De Coster $^{1}$, Damien Loterie ${ }^{1}$, Jürgen Van $\operatorname{Erps}^{1}$, Michael Vervaeke ${ }^{1}$, Jeroen \\ Missinne $^{2}$, Hugo Thienpont ${ }^{1}$, Heidi Ottevaere ${ }^{1}$ \\ ${ }^{1}$ Vrije Universiteit Brussel, Department of Applied Physics and Photonics, Brussels Photonics Team, \\ Pleinlaan 2, B-1050 Brussels, Belgium \\ ${ }^{2}$ Centre for Microsystems Technology (CMST), Imec and Ghent University, Technologiepark \\ 914A, B-9052 Gent, Belgium
}

\begin{abstract}
Raman spectroscopy is a powerful optical and non-destructive technique and a well-known method for analysis purposes, especially to determine the molecular fingerprint of substances. Traditionally, such analyses are done in a specialized lab, with considerable requirements in terms of equipment, time and manual sampling of substances of interest. In this paper we take a step from bulky Raman spectroscopy laboratory analyses towards lab-on-chip (LOC) analyses. We present an optofluidic lab-on-chip for confocal Raman spectroscopy, which can be used for the analysis of liquids. The confocal detection suppresses the unwanted background from the polymer material out of which the chip is fabricated. We design the free-form optical reflector using non-sequential ray-tracing combined with a mathematical code to simulate the Raman scattering behavior of the substance under test. We prototype the device in Polymethyl methacrylate (PMMA) by means of ultraprecision diamond tooling. In a proof-of-concept demonstration, we first show the confocal behavior of our Raman lab-on-chip system by measuring the Raman spectrum of ethanol. In a next step, we compare the Raman spectra measured in our lab-on-chip with spectra measured with a commercial Raman spectrometer. Finally, to calibrate the system we perform Raman measurements on urea solutions with different concentrations. We achieve a detection limit that corresponds to a noise equivalent concentration of $20 \mathrm{mM}$. Apart from strongly reducing the background perturbations, our confocal Raman spectroscopy system has other advantages as well. The reflector design is robust from a mechanical point of view and has the potential for mass-manufacturing using hot embossing or injection molding.
\end{abstract}

Keywords: Raman spectroscopy, optics, polymer, microfluidic, confocal detection

\section{INTRODUCTION}

Chemical analyses of components and substances in fluids are of importance in application fields such as medicine, life science, agriculture and the pharmaceutical industry. However one needs sensitive techniques to detect and quantify the limited amount of molecules that are present in the sample. One of the optical detection techniques that is used is Raman spectroscopy, a non-invasive, label-free technology utilized in a wide range of applications, including biomedical diagnostics ${ }^{[1][2]}$, inorganics and minerals examination ${ }^{[3][4]}$, quantitative polymer studies ${ }^{[5]}$, solid phase organic chemistry ${ }^{[6]}$, pharmaceutical research ${ }^{[7]}$. The traditional Raman spectroscopy analyses are done in a specialized lab, with considerable requirements in terms of equipment, time and manual sampling of the substances of interest. More and more there is a trend to use labs-on-chip (LOC) and reduce the cost and complexity of the bulky laboratory analyses by miniaturizing and integrating multiple laboratory processes on a single device.

The implementation of LOC devices for fluidic detection has the potential to overcome the limitations of the traditional Raman spectroscopy with background from the substrate and lack of portability, and hence it becomes an attractive field recently. The main design concerns when developing a Raman lab-on-chip system are the quality of the detected signal and the Raman signal collection efficiency of the system. A good signal quality means that sources of background signal and distortions must be minimized to preserve the Raman signature of the substance of interest. The signal collection efficiency of the system is important for lab-on-chip applications, because this affects the speed and the sensitivity of the system. Since the intensity of Raman scattering is very low, the Raman signal can be easily obscured by the fluorescence of the sample, stray light and parasitic Raman signals from the material of the chip. In the context of Raman spectrometer, various approaches are utilized to solve the above problems. The use of near-infrared (NIR) wavelength excitation can

Micro-Optics 2016, edited by Hugo Thienpont, Jürgen Mohr, Hans Zappe,

Hirochika Nakajima, Proc. of SPIE Vol. 9888, 98880E · C) 2016 SPIE

CCC code: $0277-786 \mathrm{X} / 16 / \$ 18 \cdot$ doi: $10.1117 / 12.2227386$

Proc. of SPIE Vol. $988898880 \mathrm{E}-1$ 
significantly reduce the fluorescence, a proper design of the optical system to limit the excitation of extraneous material beyond the sample can eliminate the background noise. Besides, multi-excitation technique ${ }^{[8]}$ and online algorithm for data processing ${ }^{[9]}$ can be applied to suppress the fluorescence and to increase the efficiency. In 2011, Ashok et al. implement a Waveguide Confined Raman Spectroscopy (WCRS) embedded fibers on a microfluidic chip ${ }^{[10]}$. They fabricated the chip in polydimethylsiloxane (PDMS) in which four channels are created using a soft lithography process. Two of the channels are embedded with multimode fibers for laser excitation and Raman signal collection respectively, the others are used as the inlet and outlet of the microfluidics. The minimum detection limit of this device for urea detection was estimated to be $80 \mathrm{mM}$ with acquisition time of $5 \mathrm{~s}$ with $200 \mathrm{~mW}$ excitation power at $785 \mathrm{~nm}$. In 2013, Dochow et al. improved a Raman-on-chip device which achieved a limit of detection up to $0.53 \mathrm{mM}$ for urea detection ${ }^{[11]}$. Unlike the microfluidic chip of Ashok's, their chip was made from quartz, which has a low Raman signature. They also increased the illumination power to $700 \mathrm{~mW}$ and the acquisition time to $10 \mathrm{~s}$.

We discuss a polymer-based lab-on-chip device embedded with a free-form reflector for confocal Raman measurements. With this novel approach we aim at enhancing the detection of Raman signals from the substance of interest due to the suppression of the parasitic Raman signals from the Polymethyl Methacrylate (PMMA) material of the chip. We ensure a robust design for the reflector chip and make us of fabrication technologies compatible with mass manufacturability, paving the way to a low-cost and disposable lab-on-chip. In section 2, we present the design of the freeform reflector and calculate the most optimum reflector shape. In section 3, we simulate the entire Raman detection setup including the excitation and collection by means of non-sequential ray-tracing combined with a mathematical code for the simulation of the Raman scattering. In section 4, we describe the fabrication of the free-form reflector and bonding of the PMMA chip while in section 5, we discuss the use of our optofluidic chip in a proof-of-concept demonstration setup for the detection of different solutions. Finally, in section 6, the conclusion is drawn for our confocal Raman spectroscopy based optofluidic chip.

\section{DESIGN OF THE INTEGRATED FREE-FORM REFLECTOR}

\subsection{Principles of confocality and the free-form reflector}

We introduce a plastic lab-on-chip equipped with a free-form reflector for confocal Raman measurements ${ }^{[12][13]}$. With this novel approach we aim at enhancing the detection of Raman signals from the substance of interest due to the suppression of the parasitic Raman signals from the PMMA material of the chip.

Confocal microscopes contain a pinhole in the optical path that rejects out-of-focus light. Fig. 1 shows such a system. Light coming from a source in the focal plane is focused by the lens to a point inside the pinhole, and all of the light can come through the pinhole. However, a light bundle originating from an out-of-focus source is not focused inside the pinhole by the lens. In this case, the bundle is too big to pass through the pinhole unattenuated. Such a confocal microscope can significantly increase the performance of a Raman spectroscopy system, even in lab-on-chips. Indeed, only the light coming from the desired location within the sample makes it to the detector. Any out-of-focus source is strongly attenuated, making the measurement practically background-free.

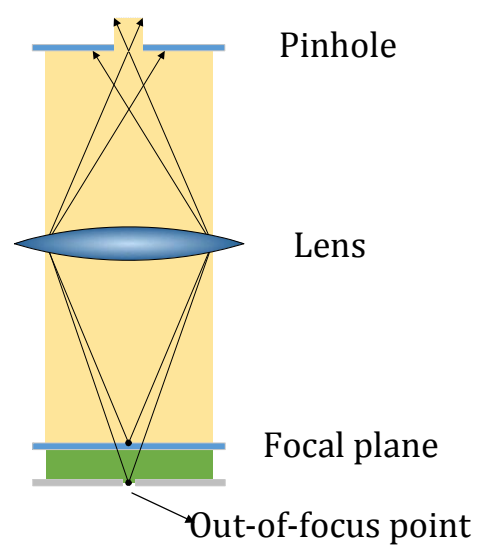

Figure 1. A confocal system rejects out-of-focus light. 
Parabolic surfaces reflect all rays parallel to the optical axis towards a focal point. Conversely, all rays originating in the focal point are collimated into a beam parallel with the optical axis. Applications include satellite dishes, telescopes and infrared microscopes. Parabolic reflectors can also be useful in a lab-on-chip context, as demonstrated by Merenda et al. in $2007^{[14]}$. In this paper, an array of more than 400 parabolic microreflectors was used to optically trap fluorescent particles in a microfluidic channel and collect the emitted fluorescence.

\subsection{Introduction of the optofluidic chip and the surrounding optics}

Our chip (shown in Fig. 2 and indicated by the dashed oval on Fig. 3) consists of three layers of PMMA. The bottom layer is a layer of $200 \mu \mathrm{m}$ thickness with a free-form optical surface on the bottom, coated with a reflective gold layer. The $500 \mu \mathrm{m}$ thick middle layer consists of a fluidic channel that runs through the focus of the aforementioned free-form reflector (Fig. 3). The use of a reflector on the bottom layer of the LOC allows a higher collection efficiency of the Raman scattered light that will be created in this focal point as compared to a design where a refractive lens is placed on the top layer. Additionally, a $500 \mu \mathrm{m}$ thickness layer is bonded on the top to sealing the fluidic channel.

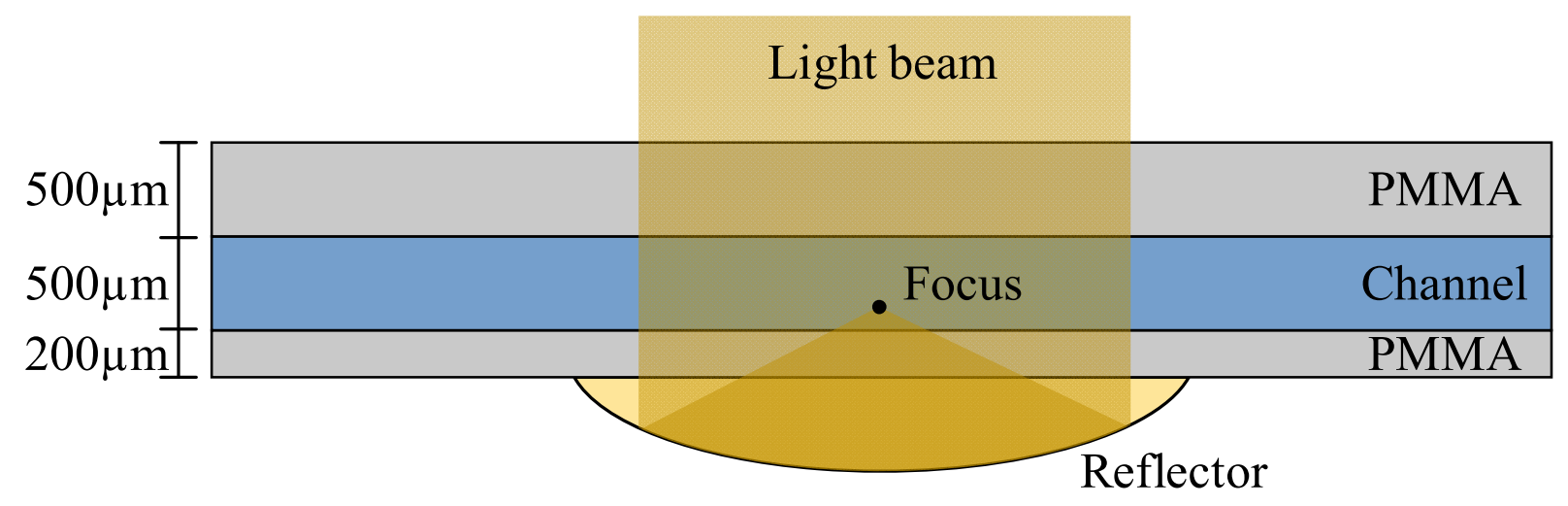

Figure 2. Geometry of Raman-on-chip that consists out of three layers.

Since our chip does not integrate the fibers for excitation and collection on the chip, external optical components are necessary (Fig.3). The divergent laser beam emitted from the excitation fiber is collimated by the lens $\mathrm{L}_{1}$. A pair of additional lenses is used to make the diameter of the laser beam smaller compared to the diameter of the reflector. The reduced beam is then reflected by the line mirror and propagates towards the chip. Due to the well optimized freeform reflector, the beam is focused to a point in the fluidic channel where the sample is. The freeform reflector also plays a role to re-collect the excited Raman scattering. Lens $\mathrm{L}_{2}$ is needed at the collection arm to focus the Raman signal into the collection fiber that is connected to the spectrometer. The notch filter in front of lens $\mathrm{L}_{2}$ can eliminate the Rayleigh scattered excitation light. In our setup, the tip of the collection fiber works as a pinhole in a normal confocal system. Indeed with the confocal geometry only the light coming from the desired location makes it to the detector. Any out-of-focus source is strongly attenuated, reducing the background in the measured signal. In the following sections the design of the reflector will be discussed theoretically. 


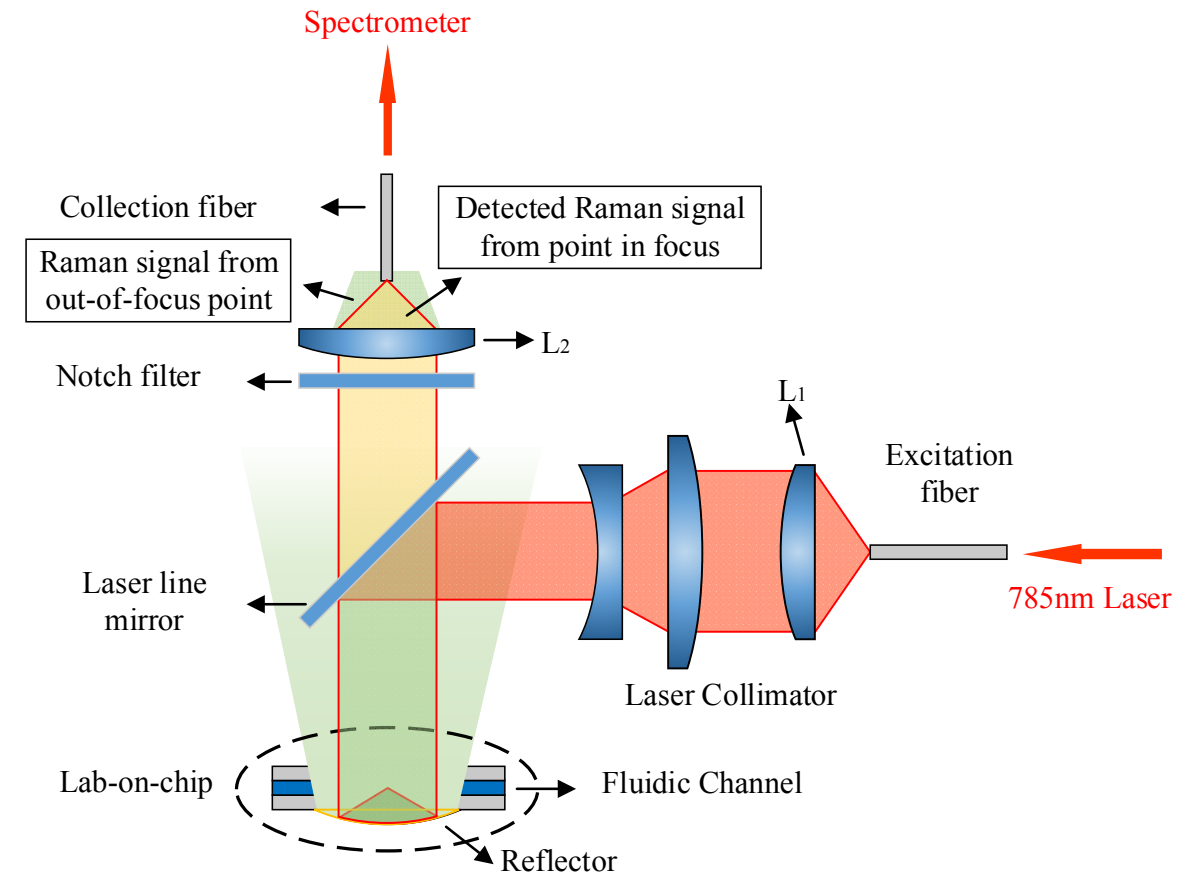

Figure 3. Simplified schematic of the full setup with a sliced view of the chip geometry. The lab-on-chip (LOC) is indicated by the dashed oval.

\subsection{Calculation of the free-form shape reflector}

The design of the reflector is based on Fermat's principle, in which we take into account the refraction of the light rays before reaching the focal point (Fig.4). In this case a parabolic reflector shape would not focus all incident light to the common focal point, especially for rays that are strongly bent which is the case due to the high NA of the reflector. This would cause significant aberrations and a loss in performance since off-axis rays are not properly focused into the desired spot. For this reason, we design a freeform reflector based on the fundamental property of a parabolic mirror. According to Fermat's principle, the optical path $\mathrm{n}_{1}\left|Q_{i} S_{i}\right|+\mathrm{n}_{2}\left|S_{i} P_{i}\right|+\mathrm{n}_{1}\left|P_{i} R_{i}\right|+\mathrm{n}_{2}\left|R_{i} F\right|$ is constant, with $\mathrm{n}_{1}$ the refractive index of water and $\mathrm{n}_{2}$ the refractive index of PMMA.

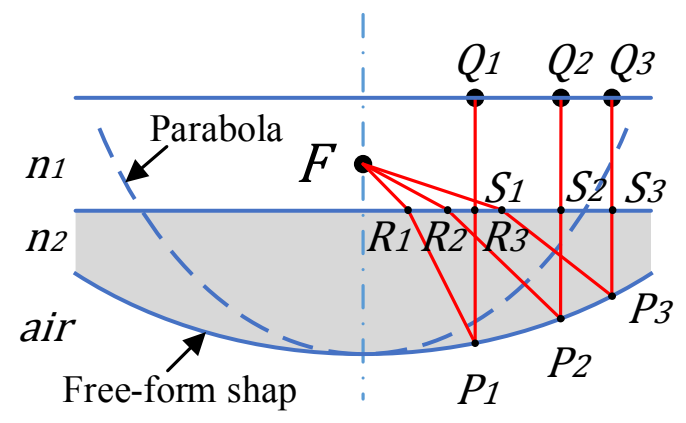

Figure 4. Derivation of the reflector's optimal shape, by accounting for a refractive transition before the focus. The medium of $\mathrm{n}_{1}$ and $\mathrm{n}_{2}$ are water and PMMA respectively. The dashed line illustrates the original parabola.

The freeform shape is calculated based on three input parameters, of which the values are chosen depending on the desired location in the microchannel for the Raman analysis and the size of the final reflector and chip. The three input parameters are the height of the reflector's focal point with respect to the bottom plate ' $h_{f}$ ', the thickness of the bottom plate ' $t_{\text {bottom }}$ ' in the chip and the maximal vertical height of the reflector ' $\mathrm{h}_{\text {reflector' }}$ ', as indicated in Fig.5. 


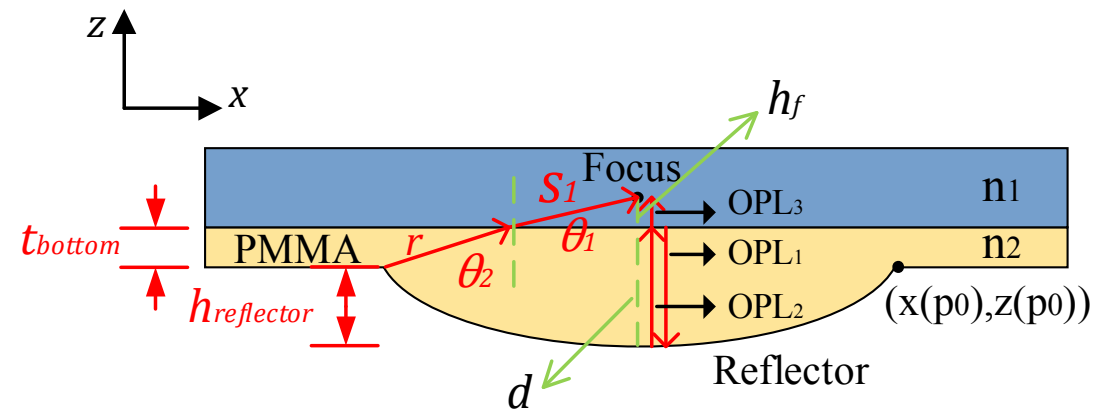

Figure 5. Parametric representation of the free-form reflector curve that focuses the parallel vertical rays in the focal point of the reflector

For modeling, we set up a parametric representation of the reflector curve. The $\mathrm{x}$ - and $\mathrm{z}$-coordinates are expressed as a function of the parameter ' $p$ '. Here ' $p$ ' is the horizontal distance of the intersection of a light ray with the water-PMMA interface of the bottom plate. $\theta_{2}$ and $\theta_{1}$ are the angle of incidence and emergence at the interface between the water and PMMA respectively. For each light ray, the distance from reflector to the water-PMMA interface is represented as ' $r$ ', the distance from the water-PMMA interface to the focus is ' $\mathrm{s}_{1}$ '. Snell's law states that:

$$
\sin \theta_{2}=\frac{\mathrm{n}_{1}}{\mathrm{n}_{2}} \sin \theta_{1}=\frac{\mathrm{n}_{1}}{\mathrm{n}_{2}} \frac{p}{\sqrt{p^{2}+\mathrm{h}_{f}^{2}}}
$$

The total constant optical path length $\left(\mathrm{s}_{t o t}\right)$ from the water-PMMA interface to the focal point is equal to

$$
\mathrm{s}_{\text {tot }}=O P L_{1}+O P L_{2}+O P L_{3}=n_{2} \cdot d+n_{2} \cdot d+n_{1} \cdot \mathrm{h}_{f}
$$

with

$$
d=\mathrm{h}_{f}+\mathrm{h}_{\text {reflector }}
$$

In this case, the optical path length travelled by each ray, from the water-PMMA interface to the focal point should equal $\mathrm{s}_{\text {tot }}$ :

$$
\begin{gathered}
s_{t o t}=n_{2} \cdot r \cdot \cos \theta_{2}+n_{2} \cdot r+s_{1} \\
r=\frac{s_{t o t}-s_{1}}{n_{2}\left(1+\cos \theta_{2}\right)}
\end{gathered}
$$

with

$$
s_{1}=n_{1} \cdot \sqrt{p^{2}+h_{f}^{2}}
$$

Finally, the $\mathrm{x}$ - and z-coordinates of the points constituting the reflector can be expressed entirely as a function of $\mathrm{p}$.

$$
\left\{\begin{array}{c}
x=p+r \cdot \sin \theta_{2} \\
z=-r \cdot \cos \theta_{2}
\end{array}\right.
$$

So the problem of refraction at the water-PMMA interface is solved numerically and resulted in a freeform shape that correctly reflects all incoming rays that are parallel to the optical axis towards the focus of the reflector. All rays originating from this focal point are then in turn collimated by the reflector when propagating out of the optofluidic chip.

Considering the fabrication process and the physical parameters of commercial PMMA materials, we chose the focal point of the reflector to be at height ' $\mathrm{h}_{f}$ ' equal to $50 \mu \mathrm{m}$ with respect to the top of the bottom layer, which has a thickness 
' $\mathrm{t}_{\text {bottom }}$ ' of $200 \mu \mathrm{m}$. In order to have a comparison, we decided to fabricate two prototypes, containing a reflector with a respective maximal height $\mathrm{h}_{\text {reflector }}$ of $300 \mu \mathrm{m}$ and $488 \mu \mathrm{m}$, corresponding to a reflector diameter of respectively $1.684 \mathrm{~mm}$ and $2.526 \mathrm{~mm}$. The effective numerical aperture (NA) of the $1.684 \mathrm{~mm}$ reflector reaches 1.28 which means the maximal incidence angle $\theta_{1}$ is $74.7^{\circ}$. To evaluate the amount of Raman scattering from the focal point collected by the reflector, we calculated the theoretical collection efficiency of the reflector, which is the percentage of light being collected from the focal point. Since there is an increased reflectivity $\mathrm{R}\left(\theta_{1}\right)$ appearing for Raman scattered light that is reaching the waterPMMA interface at large angles $\theta_{1}$, the collection efficiency can be calculated as:

$$
\operatorname{Eff}_{t h}^{r e f l}(\%)=\frac{2 \pi}{4 \pi} \cdot \int_{0}^{\theta_{1}^{\max }} \sin \theta_{1}\left(1-R\left(\theta_{1}\right)\right) d \theta_{1}=42.23 \%
$$

The collection efficiency of the $2.526 \mathrm{~mm}$ diameter reflector can be calculated by the same formula. Even though the $2.526 \mathrm{~mm}$ diameter reflector is larger than the $1.684 \mathrm{~mm}$ diameter reflector for the same focus height in the fluidic channel and bottom layer thickness of the chip, the effective NA is the same for both diameter reflectors $(\mathrm{NA}=1.28)$ since the transmitted light intensity for large angles $\theta_{1}$ is negligible due to the high reflectance $\mathrm{R}\left(\theta_{1}\right)$. However, the collection efficiency of the Raman scattering in the $2.526 \mathrm{~mm}$ diameter reflector is slightly larger ( $44 \%$ instead of $42.23 \%$ ).

\section{NON-SEQUENTIAL RAY-TRACING SIMULATIONS}

In this paper we concentrate for the optical simulations on the $1.684 \mathrm{~mm}$ diameter reflector chip, however the same reasoning can be applied to the $2.526 \mathrm{~mm}$ diameter reflector chip.

\subsection{Excitation of the Raman scattering}

In the simulation, the excitation source is a collimated Gaussian beam with a mode field diameter that is approximately half the diameter of the reflector (diameter $=1.684 \mathrm{~mm}$ ) to guarantee that a maximum amount of the incident light is reflected by the reflector and to minimize the loss of signal when the chip is misaligned with respect to the external optics. The simulated Gaussian beam starts $500 \mu \mathrm{m}$ away from the top of the chip, with a flux of 1 unit. Along its propagation, the beam creates a series of Raman scattering sources. To determine the spatial distribution of the Raman scattering, we model and design our chip by means of non-sequential ray-tracing program (ASAP). The program can record the flux that passes through a given volume element in space, but this method fails at high numerical apertures. In this case, ASAP is firstly used to trace the excitation beam until a certain distance before the focal point of the system. After that, the data of those rays including their position, direction and flux are exported to MATLAB, where we calculate the generated Raman scattering. We define Raman scattering sources along each ray of the excitation beam within the detection volume. These positions of the Raman sources are randomly selected according to a Poisson distribution. The flux of each Raman source corresponds to a scattering coefficient $\mu=1 \mu \mathrm{m}^{-1}$ which means a total amount of 1 unit flux will generate on average 1 unit of Raman scattering per micrometer. The generated Raman sources are then imported back into ASAP where the nonsequential ray-tracing continues. The rays from the laser source and the reflector are shown in fig.6 (a), whereas the resulting Raman scattering around the focus is shown in fig.6 (b). These simulations show that our approach corresponds well to the full non-sequential ray-tracing method.

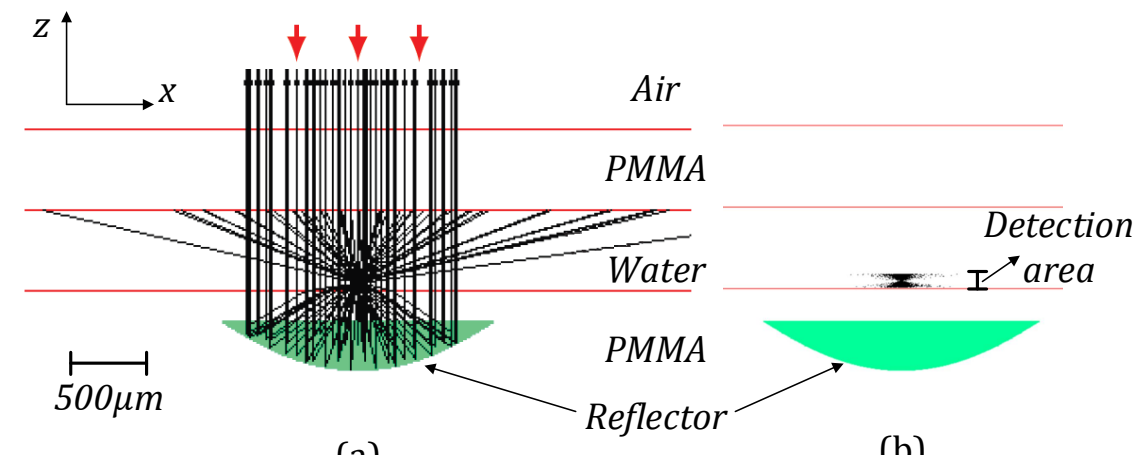

(a)

(b)

Figure 6. Excitation (a) and creation of the Raman scattering in the microfluidic channel (b) by non-sequential ray-tracing 


\subsection{Collection of the Raman scattering}

The collection arm in our design contains a notch filter, a collection lens $\mathrm{L}_{2}$, a multimode fiber and the spectrometer's slit. We also present the simulations using non-sequential ray tracing in ASAP (Fig.7). A theoretical light emitting point source in the focal point of the embedded reflector on the chip is created as Raman scattering source. The light beam reflected and collimated by the reflector is coupled into a $200 \mu \mathrm{m}$ diameter core multimode fiber with NA $=0.22$ by a collection lens with NA $=0.16$ (Thorlabs C260TME-B). The rays that reach the entrance of the collection fiber are then propagated through the fiber (simulated by a glass rod with $\mathrm{n}_{\text {core }}=1.45$ and $\mathrm{n}_{\text {cladding }}=1.4$ ) and though the spectrometer's slit with NA=0.07. Since the excitation Raman source is not one detection 'point', a detection area should be considered as the Raman scattering will occur in the area around the focal point excited by the excitation beam. By nonsequential ray tracing we can record the generated Raman scattering and detected power units from different extents of the Raman scattering area L taking into account the out-of-focus Raman sources (Table.1). We can clearly see that the spectrometer is an important bottleneck in the measurement chain.

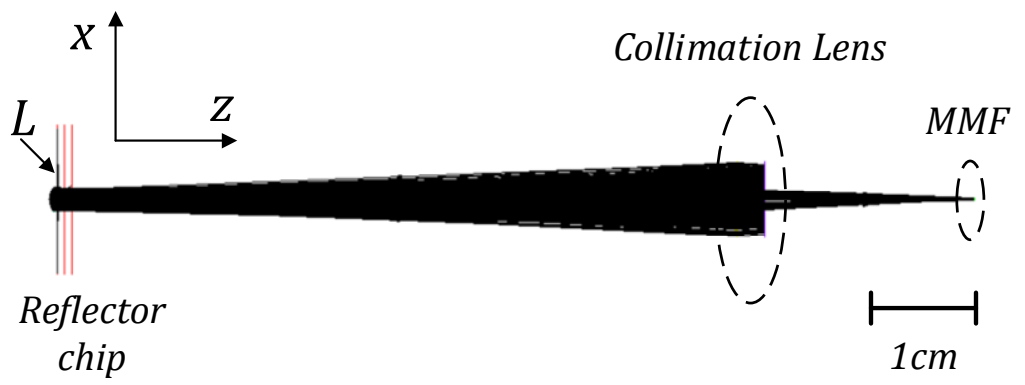

Figure 7. Simulation of the collection of Raman scattered light

Table 1. Generated Raman scattering and detected power units for different extents of the Raman scattering area L.

\begin{tabular}{|l|l|l|l|l|}
\hline $\mathrm{L}$ & Raman scattering & Input MM fiber & Output MM fiber & After slit \\
\hline $20 \mu \mathrm{m}$ & 25.56 units & 3.73 units & 3.48 units & 0.74 units \\
\hline $60 \mu \mathrm{m}$ & 76.69 units & 3.96 units & 3.69 units & 0.78 units \\
\hline $80 \mu \mathrm{m}$ & 102.26 units & 3.99 units & 3.72 units & 0.79 units \\
\hline
\end{tabular}

\section{FABRICATION OF THE LAB-ON-CHIP WITH INTEGRATED REFLECTOR}

\subsection{Free-form shape manufacturing and gold coating}

The free-form shape of the reflector is manufactured in PMMA (Hesaglas Notz Plastics AG) with in-house ultraprecision diamond tooling (Nanotech 350FG). The used diamond cutting tool has a radius of $221.3 \mu \mathrm{m}$. In the next step, the manufactured reflector is characterized with a non-contact optical surface profiler (WYKO NT2000, Veeco Inc.) to determine the surface roughness (root mean square (RMS) roughness $\mathrm{R}_{q}$ and average roughness $\mathrm{R}_{a}$ ). The surface roughness measurement results of the $1.684 \mathrm{~mm}$ and $2.526 \mathrm{~mm}$ diameter reflector are shown in Table 2. After the measurements the reflectors are coated with a 200nm-thick gold layer using a thermal evaporation process (at the 'Centre for Microsystems Technology' (CMST), Ghent University) to ensure maximal reflectance at $785 \mathrm{~nm}$ and higher wavelengths.

Table 2. Surface roughness measurement result, measured by averaging 22 randomly-selected evaluation areas of $45 \mu \mathrm{m} \times 64 \mu \mathrm{m}$ on both reflector surfaces.

\begin{tabular}{|l|l|l|}
\hline Reflector diameter & \multicolumn{1}{|c|}{$\mathrm{R}_{q}$} & \multicolumn{1}{c|}{$\mathrm{R}_{a}$} \\
\hline $1.684 \mathrm{~mm}$ & $(8.97 \pm 1.24) \mathrm{nm}$ & $(7.05 \pm 0.99) \mathrm{nm}$ \\
\hline $2.526 \mathrm{~mm}$ & $(12.38 \pm 4.3) \mathrm{nm}$ & $(9.63 \pm 3.37) \mathrm{nm}$ \\
\hline
\end{tabular}




\subsection{Bonding of the PMMA chip layers and the in- and outlet connections}

As described above, the lab-on-chip consists out of three parts, the top layer, the middle layer and the bottom layer. The middle layer contains the fluidic channel that has a width of $1 \mathrm{~mm}$ and a height of $500 \mu \mathrm{m}$. Halfway the channel we have foreseen a chamber which is approximately $0.2 \mathrm{~mm}$ larger than each reflector. In the bottom layer two holes $(\varnothing=$ $1 \mathrm{~mm}$ ) were milled for the in- and outlet of the fluid. The three PMMA plates are then bonded by using UV curing adhesive, which is a fluorescent free, slightly flexible, UV/ visible adhesive with a low viscosity and which is excellent suited for bonding thermoplastics. In order to inject the samples into the channel, we made PMMA cubic connectors which connect the micro-capillary and the in- and outlet of chip together. We verified that the final fluidic channel is leak tight by delivering water into the channel with micro-capillaries. The final chips, containing the three PMMA layers and the connecters, is shown in Fig.8.

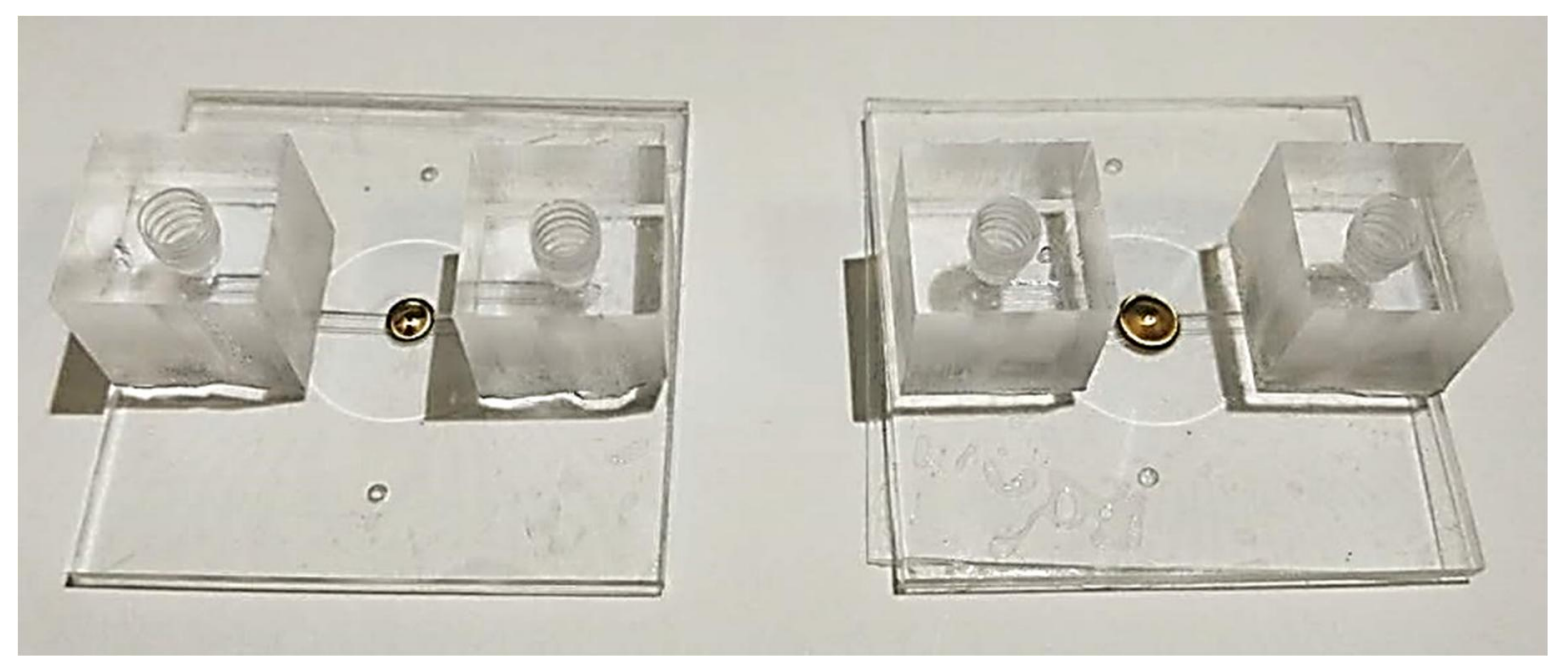

Figure 8 . Picture of the $1.684 \mathrm{~mm}$ diameter chip (left) and $2.526 \mathrm{~mm}$ diameter reflector chip (right)

\section{PROOF-OF-CONCEPT DEMONSTRATION}

In a proof-of-concept demonstration, we use a $785 \mathrm{~nm}$ wavelength laser as the Raman excitation. A single-mode optical fiber (with $\emptyset_{\text {core }}=5 \mu \mathrm{m}, \mathrm{NA}=0.12$ ) fixed with a kinematic mount at the output of the optical fiber is used and coupled with a collimator to collimate the excitation beam. A pair of lenses in front of the collimator is used to reduce the diameter of the excitation beam so that all the power of the laser can be collected by the reflector. We also introduce a laser line clean-up filter to suppress the side lobes in the spectrum of the excitation light. The beam is directed towards the optical chip via a long pass dichroic filter (pass band 790-1050nm, reflection band 784-786nm) and is reflected by the on-chip free-form reflector towards the focal point in the fluidic channel. The Raman scattering generated inside the channel is reflected by the free-form reflector and propagates through that same dichroic filter and notch filter before it is focused into the multimode fiber connected to the spectrometer. A multimode optical fiber with a core diameter of $200 \mu \mathrm{m}$ is used to collect the Raman signal. The spectrometer for the Raman spectroscopy measurements is configured for operation from $780 \mathrm{~nm}$ to $930 \mathrm{~nm}$ (a range of $2000^{-1}$ ) and comes with a slit NA of 0.07 . The FWHM resolution of the spectrometer is approximately $5 \mathrm{~cm}^{-1}$. A Peltier-cooling module is fixed inside the spectrometer to enhance its sensitivity and stability. The reflector chip is clamped on a xyz-translation stage so that we can optimize its position relative to the incident beam. The setup is illustrated in Fig.9. 


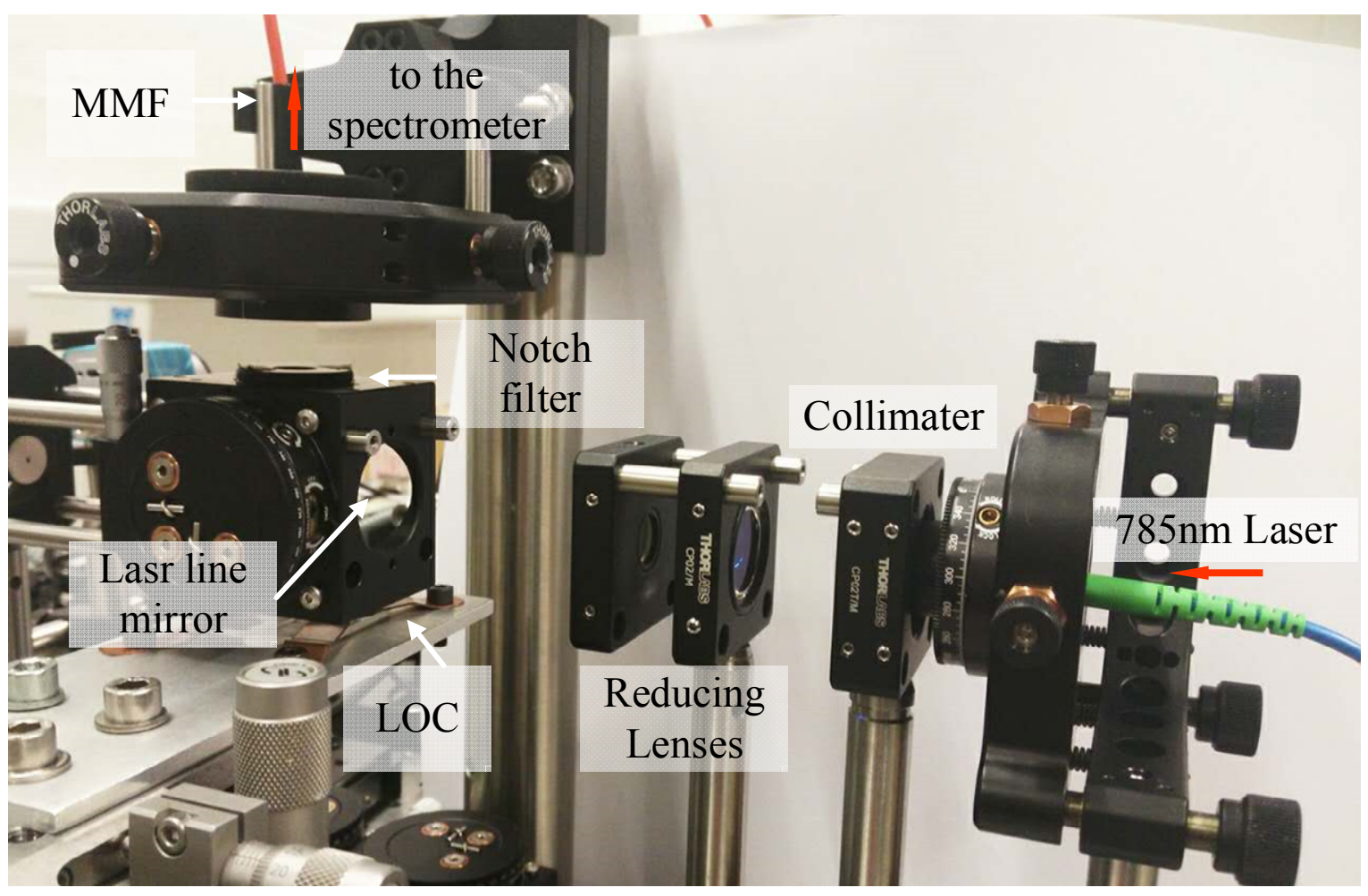

Figure 9. Proof-of-concept setup of the confocal Raman spectroscopy system

For the proof-of-concept demonstration, we prepared a set of urea solution with different molar concentrations from $450 \mathrm{mM}$ down to $20 \mathrm{mM}$ to ascertain the detection limit of our system. The urea solutions are blended from a $500 \mathrm{mM}$ urea solution with deionized water volumetrically. We calculate the accumulated errors on each concentration during this volumetric process and include these in our calibration curve. To insert the urea solutions in the fluidic channel for analysis, we fix one end of a fused silica capillary in the inlet connection on the chip. The other end is connected to a syringe, mounted on a syringe pump.

\section{RESULT AND DISCUSSION}

In Fig.10, the dash-dotted line represents the Raman spectrum of PMMA, the solid line represents the Raman spectrum of ethanol. Both spectra are measured by a commercial Raman spectrometer ('LabRAM HR Evolution' (HORIBA Scientific) for which the most prominent peaks of the respective materials in the considered range are indicated. Normally when exciting the same power, the generated Raman peak of PMMA at $800 \mathrm{~cm}^{-1}$ is higher than that of ethanol at $884 \mathrm{~cm}^{-1}$. However, thanks to our confocal design, the PMMA background in the ethanol Raman spectrum measured by our chips at $785 \mathrm{~nm}$ is suppressed by a factor up to 7 compared to the Raman spectrum of PMMA measured by the commercial Raman spectrometer. Fig. 11 shows the Raman spectra of ethanol measured by our $2.526 \mathrm{~mm}$ and $1.684 \mathrm{~mm}$ diameter lab-on-chips with an excitation beam of $190 \mathrm{~mW}$. The performance of the $2.526 \mathrm{~mm}$ diameter chip is slightly better than that of the $1.684 \mathrm{~mm}$ diameter chip, which can be explained by its higher calculated collection efficiency. We also measured the Raman signal of methanol and compared it with the Raman spectrum of ethanol (Fig.12). The different patterns of Raman spectra reveal the diversity of the internal modes between the ethanol and methanol molecules. 


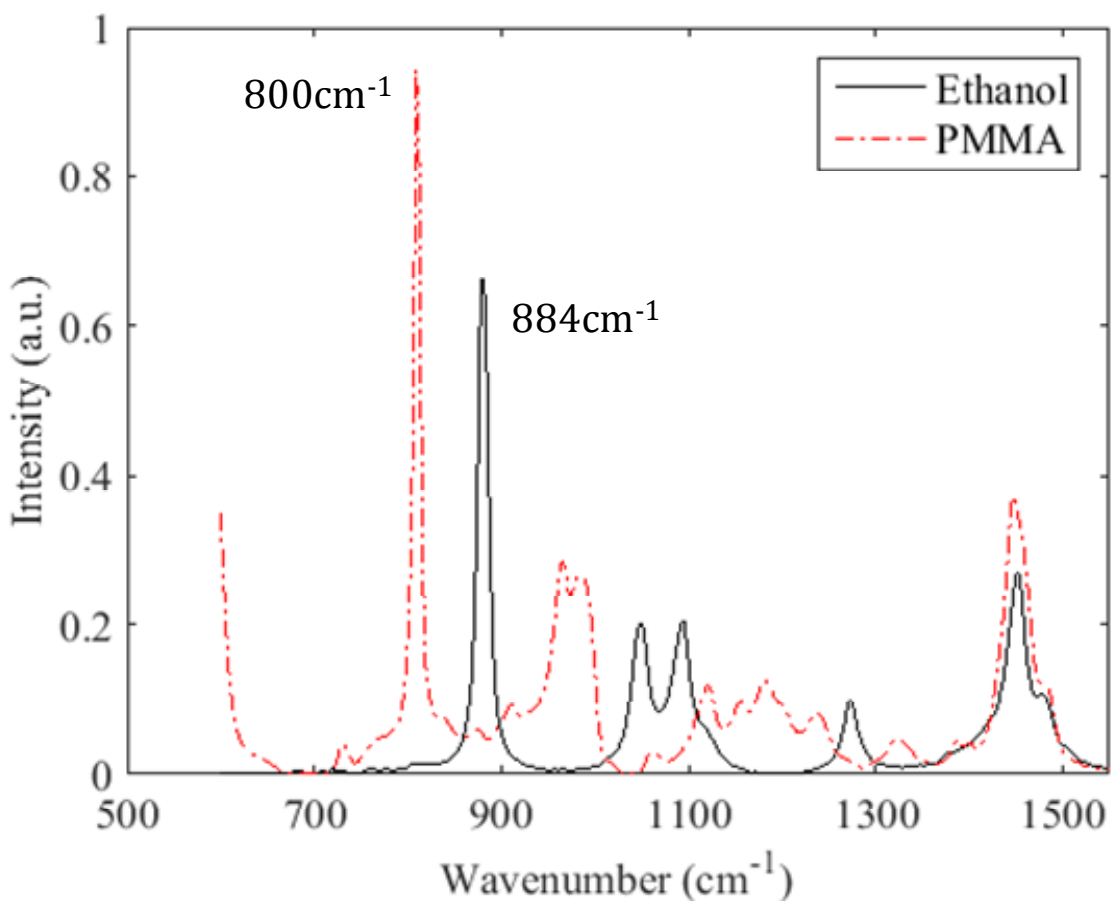

Figure 10. Raman spectra of PMMA and ethanol, measured separately with a commercial Raman spectrometer at $633 \mathrm{~nm}$

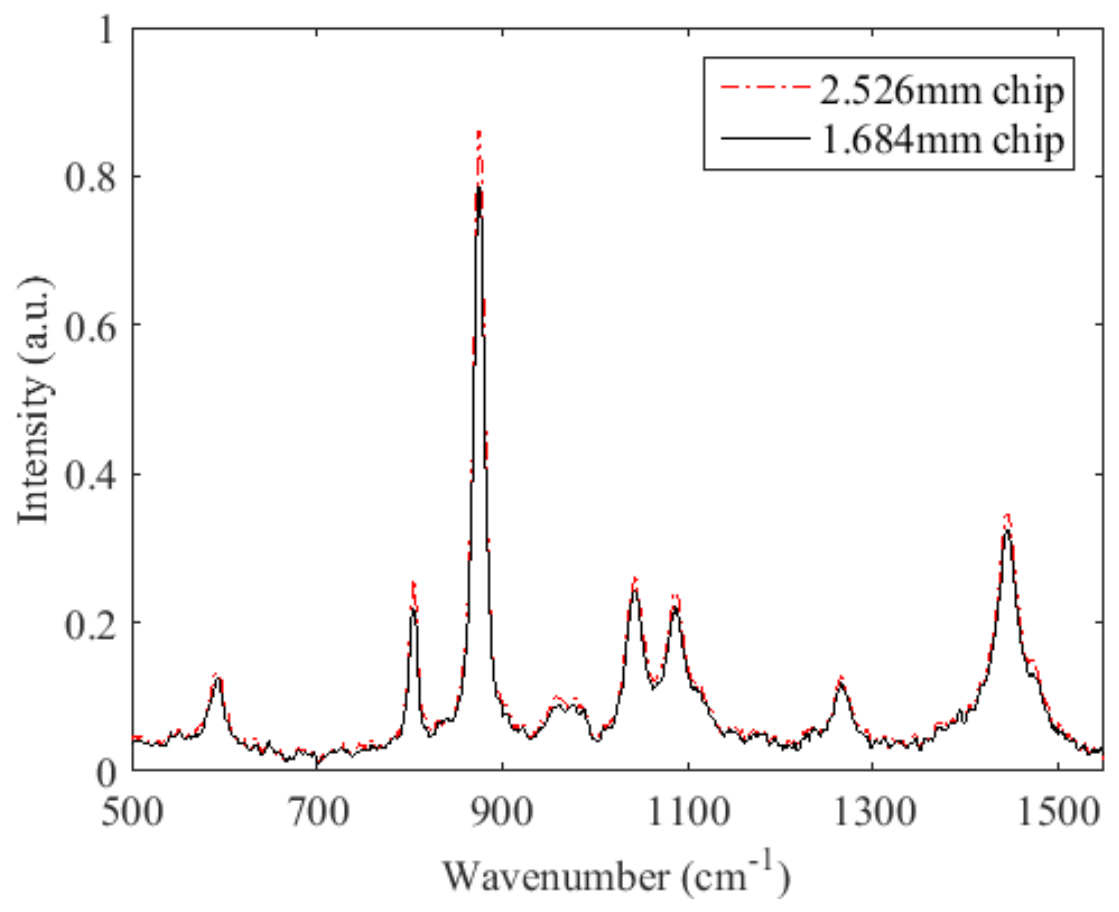

Figure 11. Raman spectra of ethanol measured by our $2.526 \mathrm{~mm}$ and $1.684 \mathrm{~mm}$ diameter lab-on-chip at $785 \mathrm{~nm}$ 


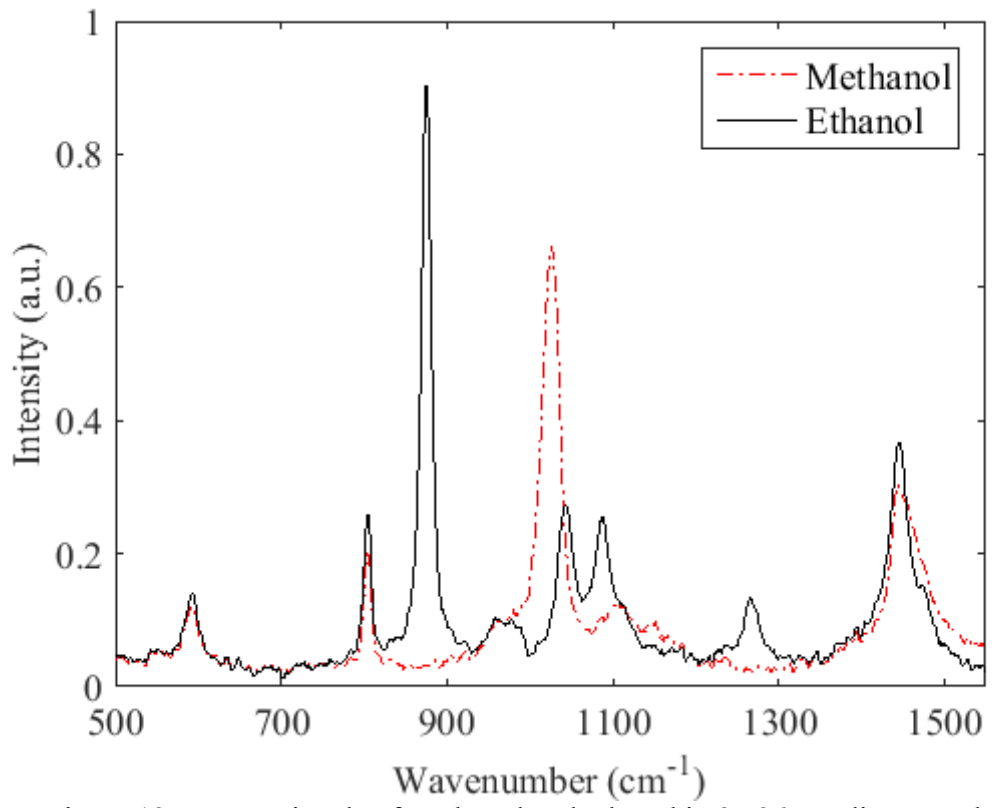

Figure 12. Raman signals of methanol and ethanol in $2.526 \mathrm{~mm}$ diameter chip

The objective of our confocal Raman system is not only to distinguish different samples qualitatively but also to analyze fluids quantitatively. To detect the limit of detection, we performed a calibration for urea dissolved in deionized water by measuring the Raman spectra of a series of urea solutions with known molar concentration using our $1.684 \mathrm{~mm}$ diameter reflector chip. The potassium nitrate (KNO3) is dissolved into the urea solutions with a constant molar concentration that acts as internal standard, since it has a strong Raman band at $1050 \mathrm{~cm}-1$ that minimally overlaps with the characteristic peak of urea (1005 cm-1). The performance of our system is characterized by means of the noise-equivalent concentration (NEC), corresponding to a SNR of 1. The SNR is calculated by dividing the mean of peak area measurements around $1005 \mathrm{~cm}-1$ by the standard deviation of these measurements. The NEC can be specified from the calibration curve shown in Fig.13. By analyzing the calibration result we can observe that the NEC of our system is $20 \mathrm{mM}$ for urea detection. The spectra are recorded with an integration time of $15 \mathrm{~s}$ and a laser excitation power of $190 \mathrm{~mW}$ at the fiber exit. The detection limit could be further lowered by shortening the collection arm in our setup or by replacing the collection arm by an adapted Raman probe, in which the excitation and collection optics would be integrated.

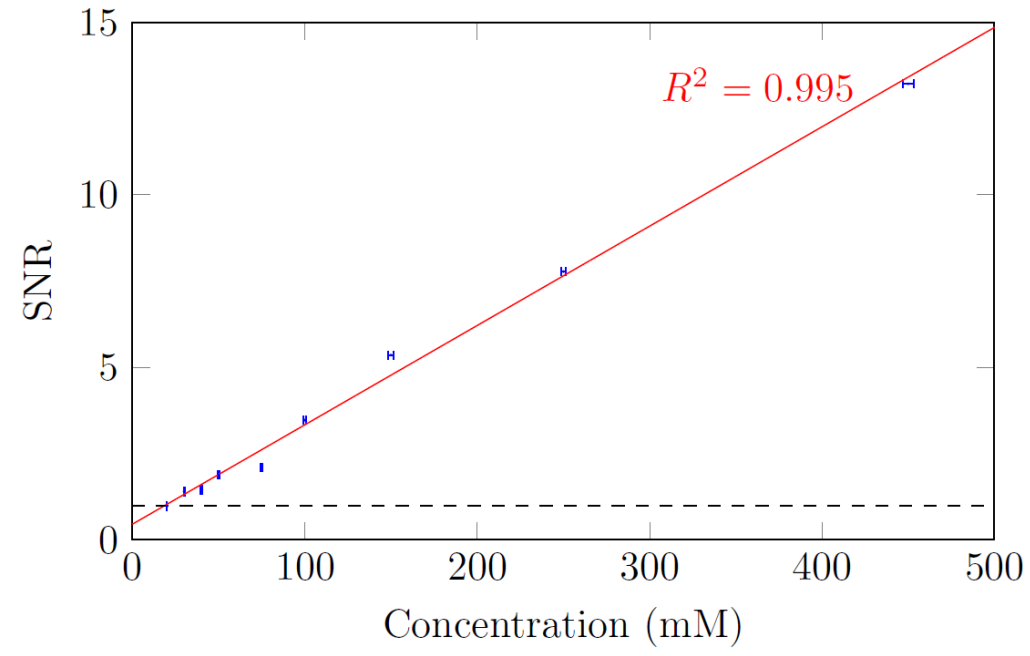

Figure 13. Calibration curve for urea measurements with an $\mathrm{R}$ squared equal to 0.995 . The dashed line represents $\mathrm{SNR}=1$, which corresponds to the noise-equivalent concentration (NEC). We achieved a NEC of $20 \mathrm{mM}$ with our system. 


\section{CONCLUSION}

We presented the design, simulation, fabrication and proof-of concept demonstration of a free-form optics enhanced optofluidic lab-on-chip for confocal Raman spectroscopy. The free-form shape reflectors of $1.684 \mathrm{~mm}$ and $2.526 \mathrm{~mm}$ diameter were designed to optimize the confocality of the system and were manufactured with in-house ultraprecision diamond tooling. During the proof-of-concept demonstration, we measured the Raman spectra of ethanol and methanol. In order to calculate the detect limitation of our lab-on-chip, we measured the spectra of urea solution from 450mM down to $20 \mathrm{mM}$ and compared the Raman signals with the internal standard (KNO3). After the calibration, we achieved a noise equivalent concentration of $20 \mathrm{mM}$ for urea detection. The experiments showed that our design can significantly suppress the background noise from the PMMA chip material with a factor of 7. Besides, the reflector designed and fabricated in PMMA makes it suitable for mass-manufacturing using hot embossing or injection molding. Finally, the use of a reflective coating enabled to achieve a very high numerical apertures $(\mathrm{NA}=1.28)$ in the microfluidic channel, increasing the collection efficiency of the confocal Raman signal. However, the use of external collection optics makes our system still bulky however there is room for further integration and miniaturization. In addition one could further improve the PMMA background suppression by optimizing the geometrical dimensions of the system. Our free-form optics enhanced confocal Raman spectroscopy for optofluidic lab-on-chip may have applications in point of drug development, care disease diagnosis, cell biology, proteomics, pharmaceutical manufacturing and environmental sensing.

\section{ACKNOWLEDGEMENT}

We acknowledge Kitty Baert and Herman Terryn of the Electrochemical and Surface Engineering group SURF (MACH) of the Vrije Universiteit Brussel (VUB) for the Raman measurements with the Horiba Scientific Raman spectrometer, Dries Rosseel for performing the CMM measurements on the reflector and Manly Callewaert for the fabrication of the in- and outlet connections.

\section{REFERENCES}

[1] Andre Downes, Alistair Elfick, "Raman Spectroscopy and Related Techniques in Biomedicine,"SENSORS, 10(3), 1871-1889 (2010)

[2] Abigail S. Haka, Karen E. Shafer-Peltier et al. "Diagnosing breast cancer by using Raman spectroscopy," Proceedings of the National Academy of Sciences of the United States of America. 102(35), 12371-12376 (2005)

[3] Changdong Shen, "Char structure characterised by Raman spectroscopy and its correlateion with combustion reactivity," FUEL, 86(15), 2316-2324 (2007)

[4] Sébastien Merkel, Alexander F. Goncharov et al. "Raman spectroscopy of Iron to 152 Gugapascals: Implicationis for Earth's Inner Core," Science, 288 (5471), 1626-1629 (2000)

[5] Shane Parnell, K. Min, M. Cakmak, "Kinetic studies of polyurethane polymerization with Raman spectroscopy," Polymer, 44 (18), 5137-5144 (2003)

[6] Michael P. Houlne, Christopher M. Sjostrom et al., "Confocal Raman Microscopy for Monitoring Chemical Reactions on single optically trapped solid-phase support particles," Anal. Chem., 74(17), 4311-4319 (2002)

[7] Giancarlo Fini, "Application of Raman spectroscopy to pharmacy," Journal of Raman Spectroscopy. 35(5), 335337 (2004)

[8] Scott T. McCain, Rebecca Willett, and David J. Brady, "Multi-excitation Raman spectroscopy technique for fluorescence rejection," Optics Express, 16(15), 10975-10991 (2008)

[9] Anna Chiara De Luca, Michael Mazilu et al., "Online Florescence Suppression in Modulated Raman Spectroscopy," Anal. Chem., 82(2), 738-745 (2010)

[10] Ashok PC et al., "Enhanced bioanalyte detection in waveguide confined Raman spectroscopy using wavelength modulatioon," Journal of Biophotonics, 4(7-8), 514-518 (2011) 
[11] Sebastian Dochow et al., "Raman-on-chip device and detection fibres Bragg grating analysis of solutions and particles," Lab Chip, 13(6), 1109-1113 (2013)

[12] T.Dieing, O. Hollricher, and J. Toporski, [Confocal Raman Micrscopy], Springer, Berling Heidelberge, Germany, 43-60 (2010)

[13] S Mozharov, A Nordon, JM Girkin, D Littlejohn, "Non-invasive analysis in micro-reactors using raman spectrometry with a specially designed probe," Lab Chip, 10, 2101-2107 (2010)

[14] Fabrice Merenda, Johann Rohner, Jean-Marc Fournier, and Rene-Paul Slathe, "Miniaturized high-NA focusingmirror multiple optical tweezers," Optics Express, 15(10), 6075-6086 (2007) 